\title{
Enhance and Protect Portfolio Returns: A Dynamic Put Spread Optimization
}

\author{
Maria Elena De Giuli ${ }^{1}$, Dennis Montagna ${ }^{1}$, Federica Naldi ${ }^{2}$ \& Alessandra Tanda ${ }^{1}$ \\ ${ }^{1}$ Department of Economics and Management, University of Pavia, Italy \\ ${ }^{2}$ Generali, Trieste, Italy \\ Correspondence: Alessandra Tanda, Department of Economics and Management, University of Pavia, Via San \\ Felice, 5, 27100 Pavia, Italy. Tel: 0039-0382-986-216. E-mail: alessandra.tanda@unipv.it
}

Received: October 3, 2019

Accepted: November 22, 2019

Online Published: November 25, 2019

doi:10.5539/ijef.v11n12p66

URL: https://doi.org/10.5539/ijef.v11n12p66

\begin{abstract}
The aim of this paper is to structure and optimize a dynamic put spread strategy to build an enhancement and protection portfolio. To implement the investment strategy a short put option acting as enhancement and a long put option providing protection are combined: the resulting put spread is modeled, thus assuming a dynamic configuration, depending on market conditions. The investment parameters and objectives are then translated into a proper optimization algorithm. The optimization procedure is implemented and backtested on S\&P500 Index as the underlying asset, and it shows that the algorithm actually results in an optimal configuration of the final put spread. The backtest additionally exhibits that the optimized strategy provides an overall over-performance with respect to the underlying asset. The paper presents a novel approach when implementing put spread strategy to enhance and protect portfolio by explicitly modeling the implied volatility and volatility skew, and dynamically adjusting the portfolio depending on market conditions.
\end{abstract}

Keywords: enhancement, implied volatility, optimization strategy, put spread, volatility skew

JEL codes: G11, G19

\section{Introduction}

The aim of this study is to structure and optimize an enhancement and protection strategy. The solution commonly identified in the literature on equity portfolio enhancement is the employment of covered call strategies as a tool to increase the risk-adjusted return of an equity portfolio (Diaz \& Kwon, 2019; He et al., 2015).

Among others, Hill et al. (2006) showed that the static covered index approach represented by the BXM (CBOE S\&P 500 Buy-Write Index), over-performs the underlying asset in low volatility environments and bear markets (on the same index, see discussion by Feldman and Roy (2005); Whaley (2002); on a different benchmark, see extensive applications by Costa et al. (2018); Kapadia and Szado (2007); Natter (2018). The same strategy (constituted by a long SPX - S\&P Index - position and a short at-the-money call) instead underperforms in high volatility environments.

Starting from the consideration that BXM outperforms on average the underlying and the performance depends on market conditions, then an alternative approach would be able to provide even more attractive risk-return trade-off than the BXM strategy proposed by Hill et al. (2006). This paper suggests a dynamic put spread solution to adjust strike prices of the options included in the portfolio to take into account the volatility environment. The components through which the final strategy is developed consist of a short put option and a long put option on the same underlying asset (i.e. an equity security), with the same time-to-maturity, but different strike prices.

More in detail, after the hint by Moreira and Muir (2017), the approach here proposed adds to the enhanced portfolio a protective tool to reduce the risk associated with the portfolio when volatility is high (on the issue, see also Cederburg et al., 2019). The strategy differs from the typically implemented long position on the underlying bought at low cost. Differently than Moreira and Muir (2017), who maintain that an optimal portfolio could be the combination of a buy-and-hold portfolio with a managed volatility portfolio, the approach of this study is to substitute the buy-and-hold component with an enhancement tool. 
In addition the strategy built with our optimization algorithm is able to overcome the high cost of the protective put approach described by Israelov and Nielsen (2015). Moreover the strategy here proposed also overcomes the issue of the underperformance of the collar strategy (see Israelov \& Klein, 2016; Israelov \& Nielsen, 2015). This paper in fact does not implement a typical collar, but using a dynamic optimization approach, it synthetically replicates the covered call through a short put option, whose optimal strike prices are individuated taking into account the volatility environment. The development of the strategy and the optimization process builds on the modelling procedure of the volatility skew as in De Giuli et al. (2019) and on the consideration of the strategic importance of volatility when building optimal portfolios (Dachraoui, 2018; Dopfel \& Ramkumar, 2013; Fallon et al., 2015; Fleming et al., 2001; Gron et al., 2011; Harvey et al., 2018; He et al., 2015; Polasek \& Pojarlev, 2004).

The paper is structured as follows: section 2 presents the methodology, comprising the strategy, the optimization procedure and the data; section 3 discusses the results; and the last section concludes.

\section{Methodology}

To reach the investors objective of enhancement and protection, a short put and a long put are used to build a strategy. The options have the same time-to-maturity but different strike prices. The put spread, resulting from the combination of the short put option and the long put option, could potentially configure as a bull put spread or as a bear put spread, depending on the relationship between the different strike prices characterizing the options. Given the enhancement objective, a bull put spread is selected, as it allows receiving a premium from the short put option that is higher than the premium paid for the long put option.

The optimization process for the definition of the final strategy depends on some parameters observed on markets and finally resulting in the optimization on variables selection represented by the strike prices of the put options used within the strategy. More in detail, the starting point for the analysis and the key element to unfold the optimization model are to be found in the implied volatility. A fundamental step in the proposed optimization process consists of exploiting and explicitly modelling the volatility skew. Starting from the relationship between the convexity of the volatility skew and the at-the-money implied volatility, the model selects the optimal strike prices to maximize the probability-weighted portfolio return.

The goodness of the algorithm is validated through a backtesting procedure on the S\&P500 Index, chosen as underlying asset.

\subsection{Optimization}

The target of the optimization problem here proposed is the probability-weighted maximum possible return for the portfolio strategy, thus including profit maximization and loss minimization concepts, whose specific components are represented in Figure 1.

A commonly used method to achieve this objective is based on the normal cumulative distribution function for returns that however requires to estimate their expected values. However, in this setting the use of the delta is the most immediate solution, as it actually exactly represents the probability of interest. Moreover, the optimization algorithm proposed is independent on the expected return of the underlying asset and this further justifies the use of delta.

\begin{tabular}{lcc}
\hline If & $K_{\text {short }}>K_{\text {long }}$ & $K_{\text {short }}<K_{\text {long }}$ \\
\cline { 2 - 3 } max profit & $\frac{p_{\text {short }}-p_{\text {long }}}{100}$ & $\frac{-K_{\text {short }}+K_{\text {long }}+p_{\text {short }}-p_{\text {long }}}{100}$ \\
probability max profit & $1-\left|\Delta_{\text {short }}\right|$ & $\left|\Delta_{\text {short }}\right|$ \\
max loss & 100 & $\frac{p_{\text {short }}-p_{\text {long }}}{100}$ \\
probability max loss & $\left|\Delta_{\text {long }}\right|$ & $\left|1-\Delta_{\text {long }}\right|$ \\
\hline
\end{tabular}

Figure 1. Maximum profit and loss, and respective probabilities computation

The expected volatility from market quotations is the starting point parameter and it is specifically represented by the at-the-money implied volatility. The optimization process then collects the expectations on the underlying asset volatility over the upcoming month from the starting date, and then proceeds searching the optimal strike price combination to set up the strategy according to the enhancement and protection goals. 


\subsection{Data}

The options referred to and used within this study are all European style options on the S\&P 500 Index with one-month time-to expiry (SPX options traditional).

The dataset used to derive the volatility skew model collects historical implied volatilities, recorded weekly on a period ranging between January 7, 2011 to November 3, 2017, and corresponding to the following strike prices: $75 \%, 85 \%, 90 \%, 95 \%, 97.5 \%, 100 \%, 102.5 \%, 105 \%, 110 \%, 115 \%, 120 \%, 125 \%$. The data are retrieved from Bloomberg.

The strike price range here considered and employed in the optimization process constitutes the typical range used by practitioners: between $95 \%$ and $105 \%$ for the short put option, and between $90 \%$ and $100 \%$ for the long put option.

With regards to backtesting, the at-the-money volatility implied by the SPX option traditional on its starting date is employed as fundamental input. Additionally, other inputs are represented by all the data available at each of the starting date of the options the investor is selling and buying within the monthly rebalancing strategy.

Furthermore, options are assumed to expire on the third Friday of every month, consistently with SPX options traditional, and even if this kind of options have an A.M. settlement, for this study and specifically for backtesting computation, the settlement is assumed to be the at the day-closing index level.

\subsection{Modeling the Volatility Skew}

Starting from the at-the-money implied volatility level the entire volatility skew curve and the implied volatility levels corresponding to the various strike prices are derived. Then, the optimization target for every possible strike price combinations is computed. The Black-Scholes formula is employed to compute premiums and deltas.

More in detail, the fundamental basis for modeling the volatility skew is the relationship between the level of at-the-money implied volatility and the convexity of the curve. On the basis of the historical at-the-money implied volatility for the various strike prices, four different classes of volatility skew are obtained, in correspondence to four specific starting levels of implied volatility (Figure 2).

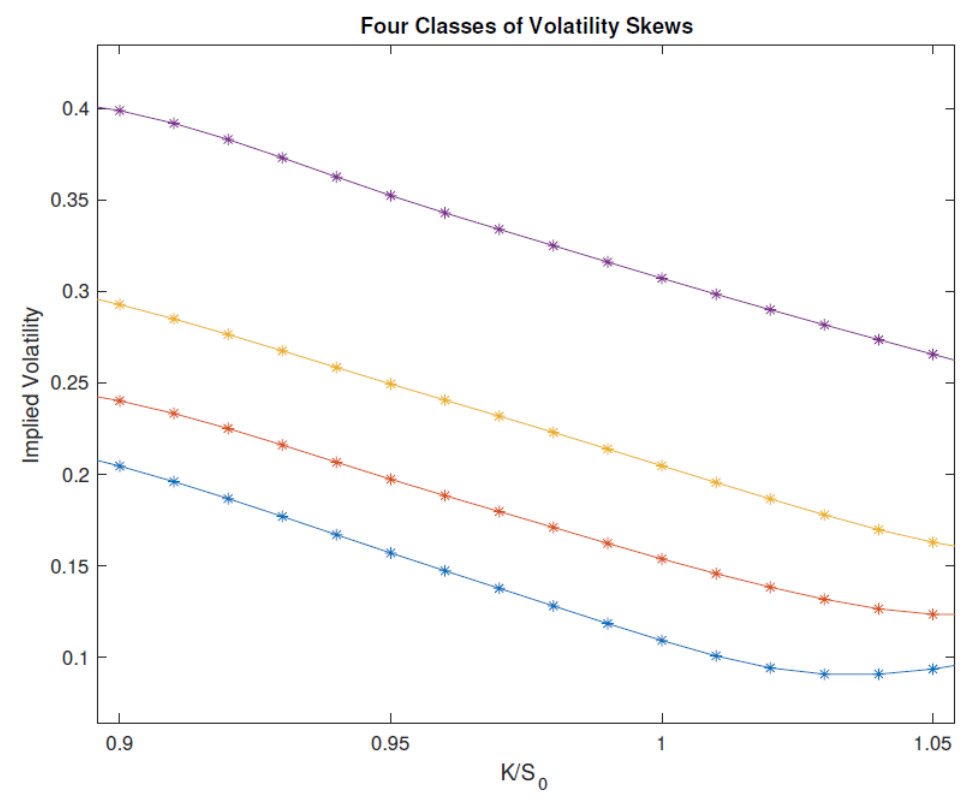

Figure 2. Four average volatility skews

To analyze the enhancement component, the target has been fixed as the probability weighted maximum profit to be maximized, while in the case of the protection component the focus is on the probability-weighted maximum loss target to be minimized. In particular, for the enhancement component, results are reported in Figure 3. Results show that with low ATM volatility the optimal strike price results to be slight ITM, consistently with high probability to have the put option expiring OTM, and so to reach the maximum profit. Instead, with high ATM volatility the optimal strike price results instead to be deep ITM, accordingly to low probability to have the 
put option expiring OTM, and so to reach the maximum profit. Furthermore, there is a consistent adjustment in terms of greeks, resulting from the optimization model (Figure 4, panel (a) and (b)).

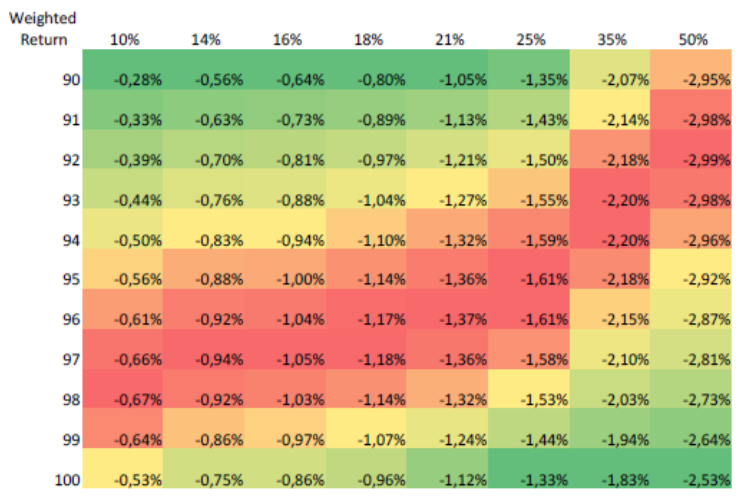

(a)

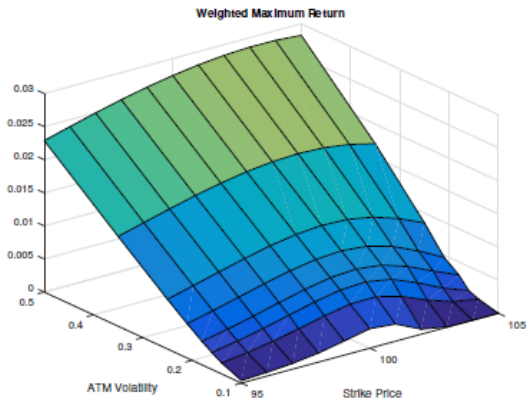

(b)

Figure 3: Enhancement component probability-weighted maximum return for four convexity classes (a) and relative surfaces $(b)$

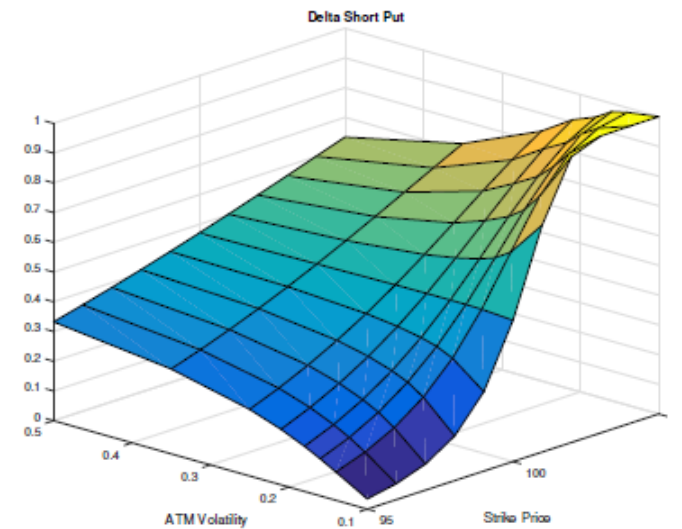

(a)

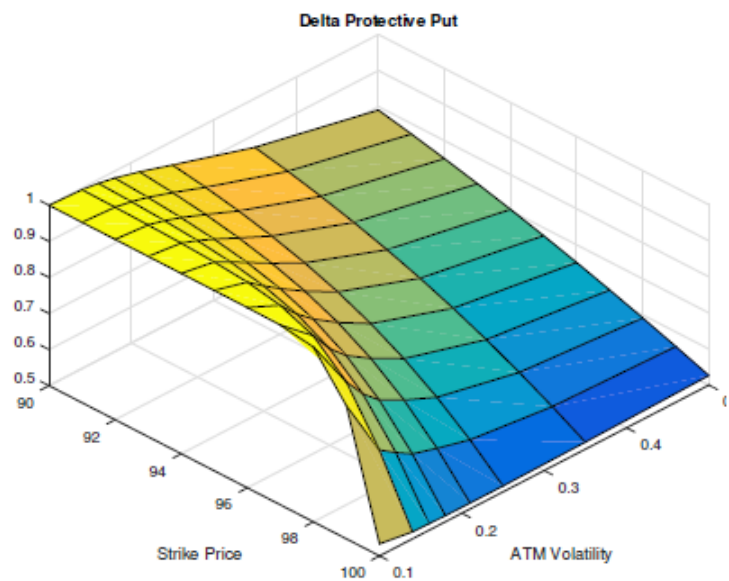

(c)

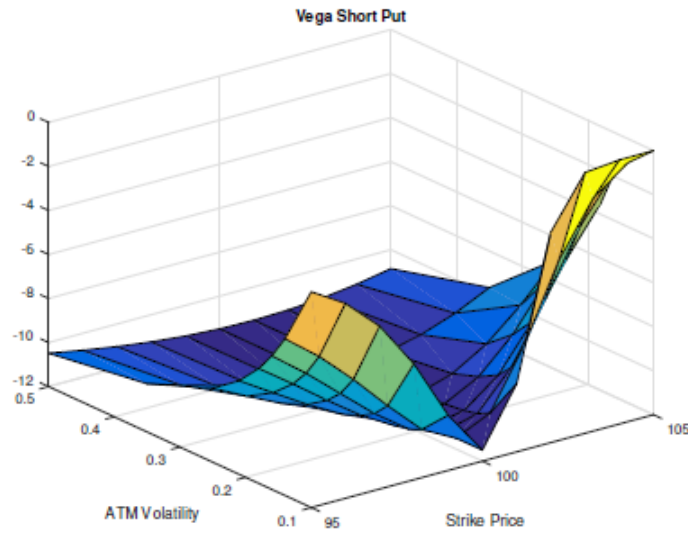

(b)

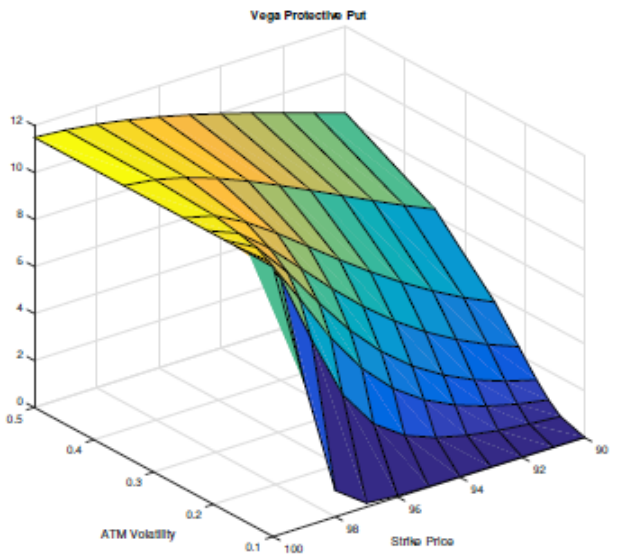

(d)

Figure 4. Delta (a) and vega (b) surfaces of short put option (enhancement component) for four convexity classes; Delta (c) and vega (d) surfaces of protective put for four convexity classes 
Referring to the protective component, represented by the long put option, the optimal strike price results to be deep OTM with low ATM volatility, while with high ATM volatility the optimal strike price configures at the ATM level, thus offering complete protection given high probability to suffer also the smallest loss (see Figure 5). Finally, a consistent adjustment in terms of greeks results from the optimization model (Figure 4, panel (c) and (d)).

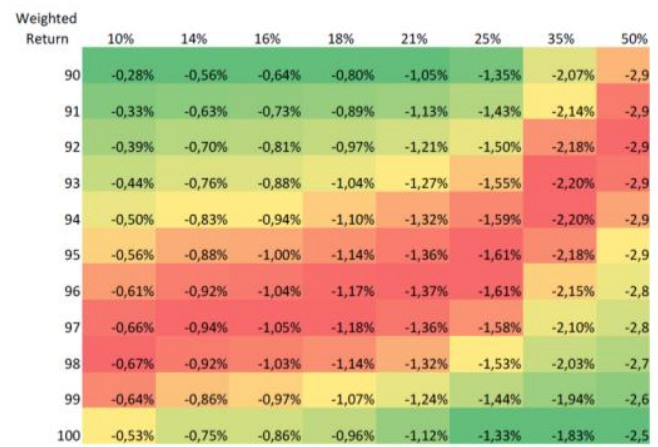

(a)

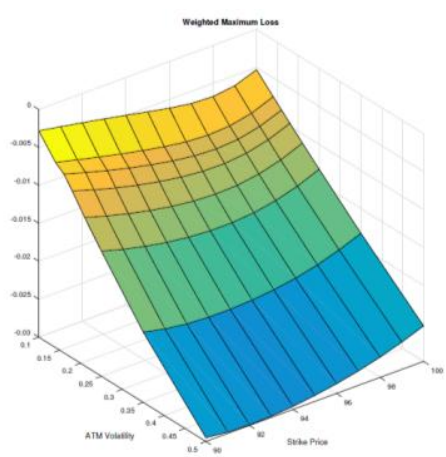

(b)

Figure 5. Protective component probability-weighted maximum loss for four convexity classes (a) and relative surface (b)

\subsection{Algorithm Building and Backtesting}

Optimization target as probability-weighted maximum possible return is defined as follows

- for $K_{\text {short }}<K_{\text {long }}$

$$
\left(\frac{-K_{\text {short }}+K_{\text {long }}+p_{\text {short }}-p_{\text {long }}}{100}\right)\left(\left|\Delta_{\text {short }}\right|\right)
$$

- $\quad$ while for $K_{\text {short }}>K_{\text {long }}$

$$
\left(\frac{\left.p_{\text {short }-p_{\text {long }}}\right)(100}{10}-\left|\Delta_{\text {short }}\right|\right)
$$

The model is translated into the final optimization algorithm as follows:

1) The ATM volatility, underlying asset price on starting date and risk-free rate are given as starting inputs for the optimization process.

2) A specific set of (three) coefficients is associated and the implied volatilities computed for the different strike prices considered.

3) The optimization target is computed in correspondence to all possible strike price combinations.

4) The strike price combination corresponding to the maximum value for the optimization target is selected.

5) Finally, the optimal strike prices as well as the corresponding implied volatility levels are returned as output.

It is important to note that the computation of the probability-weighted maximum profit is included also for short strike prices lower than long strike prices, and that consequently even these possible kind of combinations are included and tested.

However, the bear put spread, using as reference an equity index as underlying asset, never results the optimal choice if the aim is to maximize probability-weighted maximum return on the basis of the possible strike price and reference implied volatility levels used: the optimal strategy reveals always to be a bull put spread, finally in line with the aim to get the initial enhancement.

Consequently, the optimal result for maximizing probability-weighted maximum return is given always by short strike price higher than long strike price (as also confirmed by the backtesting procedure).

The algorithm backtest for optimal configuration of the strategy turns out to provide the following results:

1) With low ATM volatility (e.g. 10\%), a slightly ITM short strike price and deepest OTM long strike price as $\mathrm{K}_{\text {short }} 102 \%, \mathrm{~K}_{\text {long }} 90 \%(\Delta 50.6 \%, \mathrm{~V}-5.6)$.

2) Then with medium-high ATM volatility (e.g. 21\%), a short strike price becoming more and more ITM with increasing underlying asset expected volatility and a long strike price which remains the lowest possible as 
$\mathrm{K}_{\text {short }} 103-105 \%$, as $\mathrm{K}_{\text {long }} 90 \%(\Delta 40.5 \%, \mathrm{~V}-2.7)$.

3) With very high ATM volatility (e.g. 35\%), the short strike price configures as the highest possible and the long strike price results to offer complete protection given the high probability to suffer losses characterizing this regime, as $\mathrm{K}_{\text {short }} 105 \%, \mathrm{~K}_{\text {long }} 100 \%(\Delta 9.5 \%$, V 0.17).

\section{Results}

The optimization algorithm on historical monthly at-the-money implied volatility levels is used to derive the one-month return and to compare this return with the matching time frame return from the long position in the underlying asset.

The results of the backtesting procedure in terms of strike prices selection are reported in the Appendix (Appendix 1). Generally, the strike prices for the short put option determined by the algorithm applied on the starting levels of volatility to test, are between $102 \%$ to $105 \%$, while the long strike prices are most of the time $90 \%$. However there are some strike prices for long put option amounting to $100 \%$ and they are the ones corresponding to higher levels of at-the-money volatility, namely between almost $27 \%$ and almost $37 \%$, that correspond to price movements happening in year 2011. This result is in line with the simulation analysis previously presented using standalone approaches.

When the at-the-money implied volatility is very high with respect to the average value, the probability to suffer a loss is high as well and so there is the need to protect also from small losses given the focus on probability-weighted maximum return. Figure 6 clearly shows the relationship between the S\&P500 Index level and the ATM implied volatility. Drops in the index level correspond to expected volatility spikes, while rises in the index level correspond to at-the-money volatility drops.

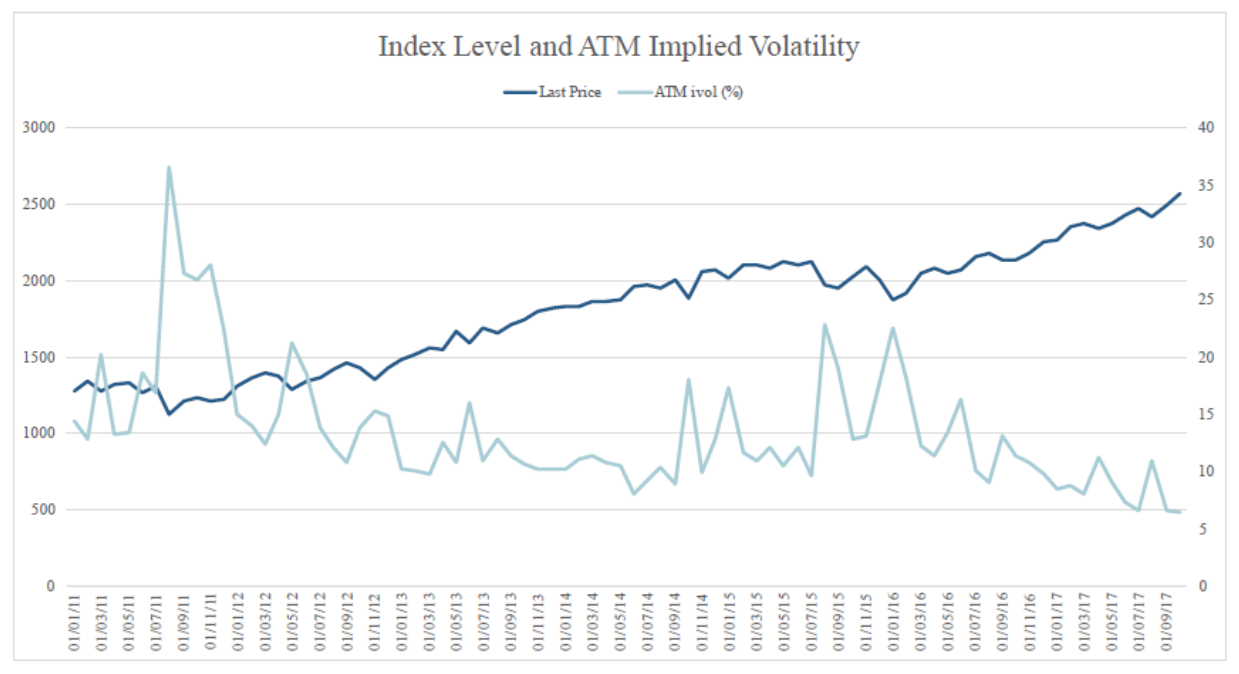

Figure 6. Index level and ATM implied volatility

In correspondence to very high levels of at-the-money implied volatility (i.e. on the 19th August, 16th September, 21 st October, and 18th November) the optimal strike prices selected by the optimization process result in a value of $100 \%$ for the long put option and a value of $105 \%$ for the short put option. For instance the highest level of at-the-money volatility coincides with the sharp drop in the index level occurring on 8th August 2011 that also affected global markets.

Furthermore, from backtesting analysis it emerges that in correspondence to those days of great volatility and low index levels, when stocks record a negative performance, the option strategy overperforms the underlying.

Moreover high levels of implied volatility correspond to an optimal strike price for the long put option of $90 \%$ while the best short strike price appears as the most in-the-money, namely the $105 \%$, and progressively falls for lower implied volatility levels.

Figure 7 specifically displays the performance results for the long position in the underlying asset and the optimized strategy. Additionally we can observe that the strategy quite follows the underlying performance but performs better in case of drops in the index levels and also to be limited in upside potential when the level of volatility drops as well. 


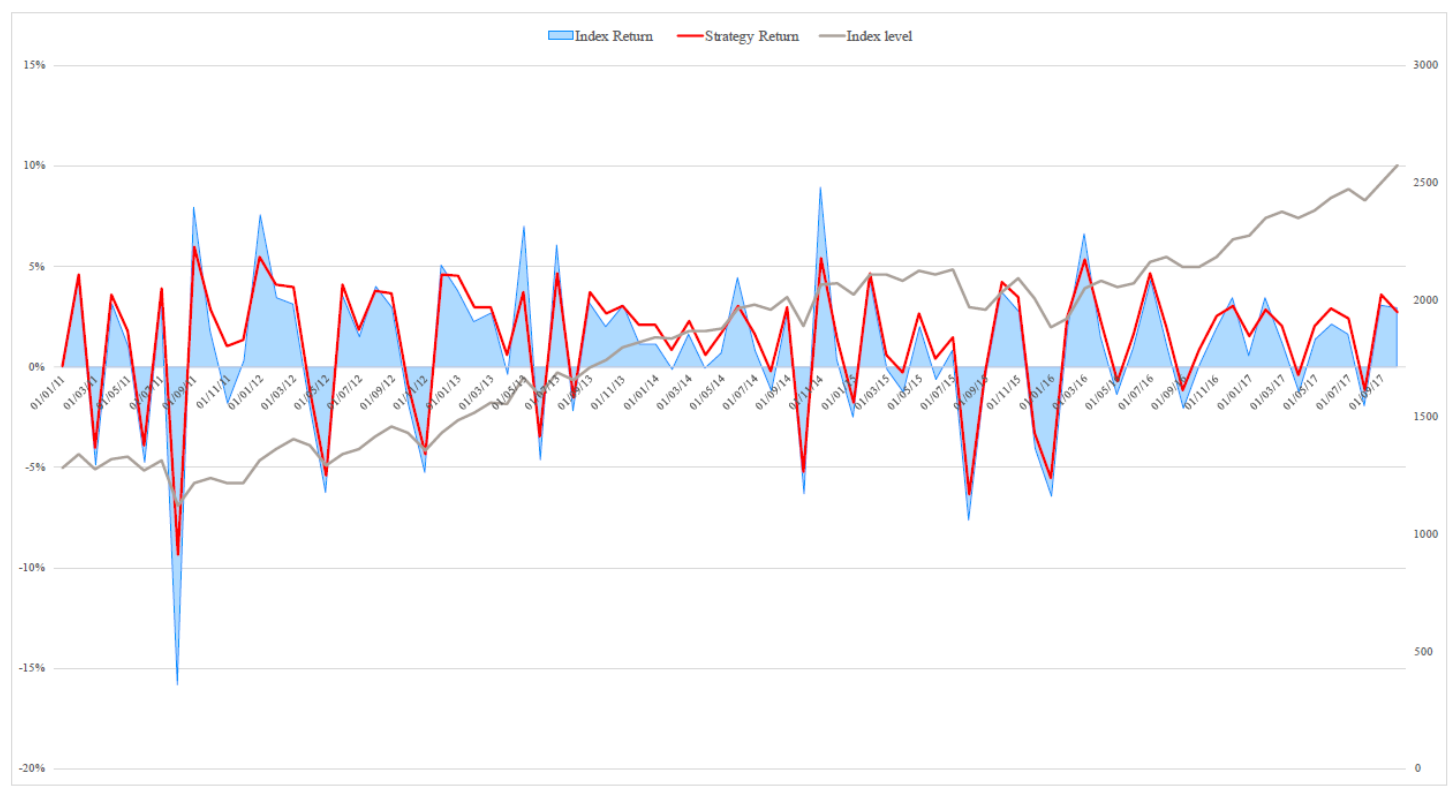

Figure 7. Returns for index, strategy and index level

Actually in some situations the long position in the index slightly over performs the strategy, typically characterized by a decrease in volatility and consequently an increase in index level realizing from the starting date of the position to the expiry date.

The underperformance occurs because the strategy proposed bounds the potential gains with respect to the long position in the underlying asset.

Despite this, the backtest shows that the strategy overall results to over-perform the long position in the index, as illustrated by performance indicator comparison reported in Figure 8. Results persist over time also in different market conditions, both in periods of high volatility (2011 crisis; second half of 2015) and low volatility (latest months of sample period).

\begin{tabular}{lcc}
\hline & Strategy & Index \\
\cline { 2 - 3 }$\mu$ & $16.2 \%$ & $10.2 \%$ \\
$\sigma$ & $10.5 \%$ & $13.3 \%$ \\
Sharpe Ratio & 1.4 & 0.7 \\
Max drawdown & $-9.3 \%$ & $-15.8 \%$ \\
Max gain & $6 \%$ & $8.9 \%$ \\
\hline
\end{tabular}

Figure 8. Performance indicators

\section{Conclusion}

This paper proposes a strategy to enhance returns and protect in case of extreme market conditions. The optimization model presented is focused on the optimal strike prices combination that is selected on the basis of at-the-money implied volatility and convexity level. The optimization target is represented by the maximization of probability-weighted maximum portfolio return.

The model proposed allows the investor to build an effective strategy, as confirmed by the positive performance results of the backtesting. Furthermore, the investor has the possibility to constantly rebalance the suggested strategy on the basis of changed expectations on volatility. Indeed, the optimization allows also to adjust the delta exposure and the vega exposure according to market conditions.

The results show that it is overall possible to over-perform the underlying asset both in terms of returns and risk-adjusted returns, both in period of low and high volatility.

In a low volatility environment, selling the put option means to bet on a further decrease in volatility: the optimal strategy will be a slightly in-the-money short strike price, since the probability to have to pay the strike price on expiry, and a deep out-the-money long strike price that would allow the investor to be protected in case of rising volatility and falling prices. 
Instead, in high volatility environment the short put option results to have a high strike price given that it is less probable that the investor will have to pay it. On the other hand, the long put option can be even configured for very high level of volatility with an at-the-money strike price in order to provide the maximum possible protection against a further increase in volatility.

\section{References}

Cederburg, S., O’Doherty, M. S., Wang, F., \& Yan, X. S. (2019). On the Performance of Volatility-Managed Portfolios. Journal of Financial Economics, Forthcoming. Retrieved from https://ssrn.com/abstract=3357038

Costa, B. A., Jakob, K., \& Niblock, S. J. (2018). Out of the Money or Striking It Rich? Evidence on the Risk-Adjusted Return Performance of Options-Based Equity Funds versus the S\& P 500 and Other Benchmark Alternatives, The Journal of Investing, 27(1), 65-79. https://doi.org/10.3905/joi.2018.27.1.065

Dachraoui, K. (2018). On the optimality of target volatility strategies. Journal of Portfolio Management, 44(5), 58-67. https://doi.org/10.3905/jpm.2018.44.5.058

De Giuli, M. E., Ligurgo, F., Montagna, D., \& Tanda A. (2019). Covered Call Strategy for yield enhancement. working paper.

Diaz, M., \& Kwon, R. H. (2019). Portfolio optimization with covered calls. Journal of Asset Management, 20(1), 38-53. DOI: $10.1057 / \mathrm{s} 41260-018-00106-0$

Dopfel, F. E., \& Ramkumar, S. R. (2013). Managed Volatility Strategies: Applications to Investment Policy. Journal of Portfolio Management, 40(1), 27-39. https://doi.org/10.3905/jpm.2013.40.1.027

Fallon, W., Park, J., \& Yu, D. (2015). Asset Allocation Implications of the Global Volatility Premium. Financial Analysts Journal, 71(5), 38-56. https://doi.org/10.2469/faj.v71.n5.4

Feldman, B. E., \& Roy, D. (2005). Passive Options-Based Investment Strategies: The Case of the CBOE S\&P 500 Buy Write Index. Journal of Investing, 14(2), 66-83. https://doi.org/10.3905/joi.2005.517177

Fleming, J., Kirby, C., \& Ostdiek, B. (2001). The Economic Value of Volatility Timing. Journal of Finance, 56(1), 329-352. DOI: https://doi.org/10.1111/0022-1082.00327

Gron, A., Jørgensen, B. N., \& Polson, N. G. (2012). Optimal portfolio choice and stochastic volatility. Applied Stochastic Models in Business and Industry, 28(1), 1-15. https://doi.org/10.1002/asmb.898

Harvey, C. R., Hoyle, E., Korgaonkar, R., Rattray, S., Sargaison, M., \& Van Hemert, O. (2018). The impact of volatility targeting. Journal of Portfolio Management, 45(1), 14-33. https://doi.org/10.3905/jpm.2018.45.1.014

He, D. X., Hsu, J. C., \& Rue, N. (2015). Option-Writing Strategies in a Low-Volatility Framework. Journal of Investing, 24(3), 116-128. https://doi.org/10.3905/joi.2015.24.3.116

Hill, J. M., Balasubramanian, V., Gregory, K., \& Tierens, I. (2006). Finding Alpha via Covered Index Writing. Financial Analysts Journal, 62(5). 29-46. https://doi.org/10.2469/faj.v62.n5.4281

Israelov, R., \& Klein, M. (2016). Risk and Return of Equity Index Collar Strategies. Journal of Alternative Investments, 19(1), 41-54. https://doi.org/10.3905/jai.2016.19.1.041

Israelov, R., \& Nielsen L. (2015). Still Not Cheap: Portfolio Protection in Calm Markets. Journal of Portfolio Management, 41(4), 108-120. https://doi.org/10.3905/jpm.2015.41.4.108

Kapadia, N., \& Szado, E. (2007). The Risk and Return Characteristics of the Buy-Write Strategy on the Russell 2000 Index. Journal of Alternative Investments, 9(4), 39-56. https://doi.org/10.3905/jai.2007.682735

Moreira, A., \& Muir T. (2017). Volatility Managed Portfolios. The Journal of Finance, 72(4), 1611-1644. https://doi.org/10.1111/jofi.12513

Natter, M. (2018). Options based benchmark indices. A review of performance and (in). appropriate measures. Journal of Futures Markets, 38(2), 271-288. https://doi.org/10.1002/fut.21865

Polasek, W., \& Pojarliev, M. (2004). Global European portfolio construction: Does a changing volatility structure matter? Applied Stochastic Models in Business and Industry, 20, 265-280. https://doi.org/10.1002/asmb.523

Whaley, R. E. (2002). Return and Risk of CBOE Buy Write Monthly Index. Journal of Derivatives, 10(2), 35-42. https://doi.org/10.3905/jod.2002.319194 


\begin{tabular}{|c|c|c|c|c|c|c|c|}
\hline Day & Last Price & $\begin{array}{l}\text { Risk Free } \\
\text { Rate(\%) }\end{array}$ & $\begin{array}{l}\text { ATM ivol } \\
(\%)\end{array}$ & $\begin{array}{l}\text { Long } \\
\text { Strike }\end{array}$ & $\begin{array}{l}\text { Short } \\
\text { Strike }\end{array}$ & $\begin{array}{l}\text { Index } \\
\text { Return }\end{array}$ & $\begin{array}{l}\text { Strategy } \\
\text { Return }\end{array}$ \\
\hline $1 / 01 / 11$ & 1283,35 & 0,15 & 14,45 & $90 \%$ & $104 \%$ & $0,00 \%$ & $0,00 \%$ \\
\hline $18 / 02 / 11$ & 1343,01 & 0,08 & 12,81 & $90 \%$ & $103 \%$ & $4,54 \%$ & $4,58 \%$ \\
\hline $18 / 03 / 11$ & 1279,20 & 0,06 & 20,26 & $90 \%$ & $105 \%$ & $-4,87 \%$ & $-4,04 \%$ \\
\hline $15 / 04 / 11$ & 1319,68 & 0,04 & 13,35 & $90 \%$ & $103 \%$ & $3,12 \%$ & $3,58 \%$ \\
\hline $20 / 05 / 11$ & 1333,27 & 0,03 & 13,47 & $90 \%$ & $103 \%$ & $1,02 \%$ & $1,77 \%$ \\
\hline $17 / 06 / 11$ & 1271,50 & 0,02 & 18,6 & $90 \%$ & $105 \%$ & $-4,74 \%$ & $-3,89 \%$ \\
\hline $15 / 07 / 11$ & 1316,14 & 0,02 & 16,87 & $90 \%$ & $104 \%$ & $3,45 \%$ & $3,89 \%$ \\
\hline 19/08/11 & 1123,53 & 0,00 & 36,69 & $100 \%$ & $105 \%$ & $-15,82 \%$ & $-9,33 \%$ \\
\hline $16 / 09 / 11$ & 1216,01 & 0,01 & 27,42 & $100 \%$ & $105 \%$ & $7,91 \%$ & $5,99 \%$ \\
\hline $21 / 10 / 11$ & 1238,25 & 0,01 & 26,72 & $100 \%$ & $105 \%$ & $1,81 \%$ & $2,84 \%$ \\
\hline $18 / 11 / 11$ & 1215,65 & 0,02 & 28,14 & $100 \%$ & $105 \%$ & $-1,84 \%$ & $1,02 \%$ \\
\hline $16 / 12 / 11$ & 1219,66 & 0,00 & 22,37 & $90 \%$ & $105 \%$ & $0,33 \%$ & $1,34 \%$ \\
\hline $20 / 01 / 12$ & 1315,38 & 0,03 & 14,97 & $90 \%$ & $104 \%$ & $7,56 \%$ & $5,47 \%$ \\
\hline $17 / 02 / 12$ & 1361,23 & 0,03 & 14,04 & $90 \%$ & $103 \%$ & $3,43 \%$ & $4,09 \%$ \\
\hline $16 / 03 / 12$ & 1404,17 & 0,07 & 12,35 & $90 \%$ & $103 \%$ & $3,11 \%$ & $3,96 \%$ \\
\hline $20 / 04 / 12$ & 1378,53 & 0,04 & 15,04 & $90 \%$ & $104 \%$ & $-1,84 \%$ & $-1,13 \%$ \\
\hline $18 / 05 / 12$ & 1295,22 & 0,06 & 21,22 & $90 \%$ & $105 \%$ & $-6,23 \%$ & $-5,44 \%$ \\
\hline $15 / 06 / 12$ & 1342,84 & 0,05 & 18,57 & $90 \%$ & $105 \%$ & $3,61 \%$ & $4,12 \%$ \\
\hline $20 / 07 / 12$ & 1362,66 & 007 & 13,82 & $90 \%$ & $103 \%$ & $1,47 \%$ & $1,86 \%$ \\
\hline $17 / 08 / 12$ & 1418,16 & 9 & 12,06 & $90 \%$ & $103 \%$ & $3,99 \%$ & $3,76 \%$ \\
\hline $21 / 09 / 12$ & 1460,15 & 0,05 & 10,79 & $90 \%$ & $102 \%$ & $2,92 \%$ & $3,64 \%$ \\
\hline $19 / 10 / 12$ & 1433,19 & 0,11 & 13,8 & $90 \%$ & $103 \%$ & $-1,86 \%$ & $-0,84 \%$ \\
\hline $16 / 11 / 12$ & 1359,88 & 0,07 & 15,33 & $90 \%$ & $104 \%$ & $-5,25 \%$ & $-4,36 \%$ \\
\hline $21 / 12 / 12$ & 1430,15 & & 100 & $90 \%$ & $104 \%$ & & $4,61 \%$ \\
\hline $18 / 01 / 13$ & 1485,98 & 4 & 10,29 & $90 \%$ & $102 \%$ & $3,83 \%$ & $4,51 \%$ \\
\hline $15 / 02 / 13$ & 1519,79 & 0,09 & 10,09 & $90 \%$ & $102 \%$ & $2,25 \%$ & $2,98 \%$ \\
\hline $15 / 03 / 13$ & 1560,70 & 0,0 & 985 & $90 \%$ & $102 \%$ & $2,66 \%$ & $2,97 \%$ \\
\hline $19 / 04 / 13$ & 1555,25 & 0 & 12 & $90 \%$ & $103 \%$ & $-0,35 \%$ & $0,61 \%$ \\
\hline $17 / 05 / 13$ & 1667,47 & 0 & 10,78 & $90 \%$ & $02 \%$ & & $3,70 \%$ \\
\hline $21 / 06 / 13$ & 1592,43 & 2 & 16,03 & $90 \%$ & $104 \%$ & $-4,60 \%$ & $-3,49 \%$ \\
\hline $19 / 07 / 13$ & 1692,09 & 0,01 & 11,03 & $90 \%$ & $103 \%$ & $6,07 \%$ & $4,64 \%$ \\
\hline $16 / 08 / 13$ & 1655,83 & 0 , & 12,79 & $90 \%$ & $103 \%$ & $-2,17 \%$ & $-1,51 \%$ \\
\hline $20 / 09 / 13$ & 1709,91 & 0,01 & 11,4 & $90 \%$ & $103 \%$ & $3,21 \%$ & $3,71 \%$ \\
\hline $18 / 10 / 13$ & 1744,50 & 0,01 & 10,68 & $90 \%$ & $102 \%$ & $2,00 \%$ & $2,67 \%$ \\
\hline $15 / 11 / 13$ & 1798,18 & 0,06 & 10,25 & $90 \%$ & $102 \%$ & $3,03 \%$ & $3,00 \%$ \\
\hline $20 / 12 / 13$ & 1818,32 & 0,01 & 10,24 & $90 \%$ & $102 \%$ & $1,11 \%$ & $2,10 \%$ \\
\hline $17 / 01 / 14$ & 1838,70 & 0,01 & 10,26 & $90 \%$ & $102 \%$ & $1,11 \%$ & $2,10 \%$ \\
\hline $21 / 02 / 14$ & 1836,25 & 000 & 11,15 & $90 \%$ & $103 \%$ & $-0,13 \%$ & $0,85 \%$ \\
\hline $21 / 03 / 14$ & 1866,52 & 0,05 & 11,45 & $90 \%$ & $103 \%$ & $1,64 \%$ & $2,29 \%$ \\
\hline $18 / 04 / 14$ & 1864,85 & 0,02 & 10,81 & $90 \%$ & $102 \%$ & $-0,09 \%$ & $0,56 \%$ \\
\hline $16 / 05 / 14$ & 1877,86 & 0,01 & 10,56 & $90 \%$ & $102 \%$ & $0,70 \%$ & $1,71 \%$ \\
\hline $20 / 06 / 14$ & 1962,87 & 0,01 & & $90 \%$ & $102 \%$ & $4,43 \%$ & $3,00 \%$ \\
\hline $18 / 07 / 14$ & 1978,22 & & 8 & $90 \%$ & $02 \%$ & $0,78 \%$ & $1,64 \%$ \\
\hline $15 / 08 / 14$ & 1955,06 & 0,03 & 10,46 & $90 \%$ & $102 \%$ & $-1,18 \%$ & $-0,25 \%$ \\
\hline $19 / 09 / 14$ & 2010,40 & 0,01 & 8,93 & $90 \%$ & $102 \%$ & $2,79 \%$ & $2,99 \%$ \\
\hline $17 / 10 / 14$ & 1886,76 & 003 & 1809 & $90 \%$ & $105 \%$ & $-6,35 \%$ & $-5,24 \%$ \\
\hline $21 / 11 / 14$ & 2063,50 & 0,04 & 9,96 & $90 \%$ & $102 \%$ & $8,95 \%$ & $5,37 \%$ \\
\hline $19 / 12 / 14$ & 2070,65 & 0,01 & 12,8 & $90 \%$ & $103 \%$ & $0,35 \%$ & $1,31 \%$ \\
\hline $16 / 01 / 15$ & 2019,42 & 002 & 17,4 & $90 \%$ & $104 \%$ & $-2,51 \%$ & $-1,76 \%$ \\
\hline $20 / 02 / 15$ & 2110,30 & 0,02 & 11,73 & $90 \%$ & $103 \%$ & $4,40 \%$ & $4,68 \%$ \\
\hline $20 / 03 / 15$ & 2108,10 & & 10,92 & $90 \%$ & $102 \%$ & $-0,10 \%$ & $0,56 \%$ \\
\hline $17 / 04 / 15$ & 2081,18 & & 2,09 & $90 \%$ & $103 \%$ & $-1,29 \%$ & $-0,26 \%$ \\
\hline $15 / 05 / 15$ & 2122,73 & 0,02 & 10,51 & $90 \%$ & $102 \%$ & $1,98 \%$ & $2,68 \%$ \\
\hline $19 / 06 / 15$ & 2109,99 & 0,00 & 12,13 & $90 \%$ & $103 \%$ & $-0,60 \%$ & $0,39 \%$ \\
\hline $17 / 07 / 15$ & 2126,64 & & 974 & 9 & 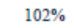 & $0,79 \%$ & $1,47 \%$ \\
\hline $21 / 08 / 15$ & 1970,89 & 0,02 & 22,89 & $90 \%$ & $105 \%$ & $-7,61 \%$ & $-6,37 \%$ \\
\hline $18 / 09 / 15$ & 1958,03 & 0,00 & 19,02 & 90 & $105 \%$ & $-0,65 \%$ & $-0,17 \%$ \\
\hline $16 / 10 / 15$ & 2033,11 & 0,03 & 12,85 & $90 \%$ & $103 \%$ & $3,76 \%$ & $4,23 \%$ \\
\hline $20 / 11 / 15$ & 2089,17 & 0,05 & 13,12 & $90 \%$ & $103 \%$ & $2,72 \%$ & $3,47 \%$ \\
\hline $18 / 12 / 15$ & 2005,55 & 0,16 & 17,78 & $90 \%$ & $104 \%$ & $-4,08 \%$ & $-3,28 \%$ \\
\hline $15 / 01 / 16$ & 1880,33 & 0 & 22,62 & $90 \%$ & $105 \%$ & $-6,45 \%$ & $-5,56 \%$ \\
\hline $19 / 02 / 16$ & 1917,78 & 0,26 & 18,24 & $90 \%$ & $105 \%$ & $1,97 \%$ & $2,46 \%$ \\
\hline $18 / 03 / 16$ & 2049,58 & 0,27 & 12,23 & $90 \%$ & $103 \%$ & $6,65 \%$ & $5,36 \%$ \\
\hline $15 / 04 / 16$ & 2080,73 & 0 & 1) & $90 \%$ & $103 \%$ & $1,51 \%$ & $2,20 \%$ \\
\hline $20 / 05 / 16$ & 2052,32 & 0,25 & 13,41 & $90 \%$ & $103 \%$ & $-1,37 \%$ & $-0,72 \%$ \\
\hline $17 / 06 / 16$ & 2071,22 & 0,22 & 16,27 & $90 \%$ & $104 \%$ & $0,92 \%$ & $1,65 \%$ \\
\hline $15 / 07 / 16$ & 2161,74 & 0,26 & 10,09 & $90 \%$ & $102 \%$ & $4,28 \%$ & $4,64 \%$ \\
\hline $19 / 08 / 16$ & 2183,87 & 0,27 & 9,04 & $90 \%$ & $102 \%$ & $1,02 \%$ & $1,98 \%$ \\
\hline $16 / 09 / 16$ & 2139,16 & 0,19 & 13,21 & $90 \%$ & $103 \%$ & $-2,07 \%$ & $-1,15 \%$ \\
\hline $21 / 10 / 16$ & 2141,16 & 0,24 & 11,47 & $90 \%$ & $103 \%$ & $0,09 \%$ & $0,82 \%$ \\
\hline $18 / 11 / 16$ & 2181,90 & 0,27 & 10,78 & $90 \%$ & $102 \%$ & $1,88 \%$ & $2,55 \%$ \\
\hline $16 / 12 / 16$ & 2258,07 & 0,46 & 9,81 & $90 \%$ & $102 \%$ & $3,43 \%$ & $3,00 \%$ \\
\hline $20 / 01 / 17$ & 2271,31 & & 8,58 & $90 \%$ & $102 \%$ & $0,58 \%$ & $1,52 \%$ \\
\hline $17 / 02 / 17$ & 2351,16 & 0,50 & 8,75 & $90 \%$ & $102 \%$ & $3,46 \%$ & $2,86 \%$ \\
\hline $17 / 03 / 17$ & 2378,25 & 0,71 & 8,09 & $90 \%$ & $102 \%$ & $1,15 \%$ & $2,02 \%$ \\
\hline $21 / 04 / 17$ & 2348,69 & 0,72 & 11,21 & $90 \%$ & $103 \%$ & $-1,25 \%$ & $-0,42 \%$ \\
\hline $19 / 05 / 17$ & 2381,73 & 0,69 & 9,06 & $90 \%$ & $102 \%$ & $1,40 \%$ & $2,01 \%$ \\
\hline /06/17 & 2433,15 & & 7,4 & 90 & 10 & 2,1 & 2,88 \\
\hline $21 / 07 / 17$ & 2472,54 & 0,98 & 6,67 & $90 \%$ & $102 \%$ & $1,61 \%$ & $2,40 \%$ \\
\hline $18 / 08 / 17$ & 2425,55 & 0,96 & 10,95 & $90 \%$ & $103 \%$ & $-1,92 \%$ & $-1,18 \%$ \\
\hline $9 / 17$ & 2500,23 & 0,97 & 6,65 & $90 \%$ & $102 \%$ & $3,03 \%$ & 3,58 \\
\hline $10 / 17$ & 2575,21 & 0,98 & 649 & $90 \%$ & $102 \%$ & $2,95 \%$ & 2770 \\
\hline
\end{tabular}

Appendix 1. ATM implied volatility, optimal strike prices and returns

\section{Copyrights}

Copyright for this article is retained by the author(s), with first publication rights granted to the journal.

This is an open-access article distributed under the terms and conditions of the Creative Commons Attribution license (http://creativecommons.org/licenses/by/4.0/). 